\title{
EMC Computer Modeling Techniques for CPU Heat Sink Simulation
}

\author{
Junwei $\mathrm{Lu}^{1}$ and Francis Dawson ${ }^{2}$ \\ ${ }^{1}$ Griffith School of Engineering, Faculty of EIT, Nathan Campus, Griffith University, Nathan, Brisbane, Qld 4111, Australia \\ ${ }^{2}$ Department of Electrical and Computer Engineering, University of Toronto, Toronto, ON M5S-3G4, Canada.
}

\begin{abstract}
This paper presents finite-element frequency domain results for electromagnetic radiation emitted from high power microelectronic circuits connected to a heat sink. The heat sink model associated with one of the IEEE EMC challenging problems has been used to investigate three different grounding configurations. A new simulation model for the Intel P4 CPU heat sink is proposed and analyzed. A resonant frequency of $2.6 \mathrm{GHz}$ with a reflection coefficient of $8.3 \mathrm{~dB}$ was found for the CPU heat sink. This is close to the IEEE and Bluetooth wireless communication system's operating frequency of $2.4 \mathrm{GHz}$. An optimal design of the CPU heat sink design should be performed in order to minimize the radiated emission from the CPU heat sink.
\end{abstract}

Index Terms-CPU heat sink, EMC, EMC challenging problem, EMC computer modeling, EMI.

\section{INTRODUCTION}

$\mathbf{M}$ ODERN silicon wafer fabrication facilities easily produce component densities that exceed 1 million transistors per die. Some components consume a significant amount of dc power. Components such as the Intel and AMD processors require separate cooling provided by a fan built into their heat sink or by a fan or cooling devices located adjacent to the processor. Since these high-power and high-speed processors are being implemented in more designs, special design techniques are now required for EMI suppression and heat removal at the component level. Obviously, full wave numerical analysis tools are required to model the radiated emissions given that traditional models are unable to do so.

In this paper we mainly consider RF domain problems that take into account the heat sink, the thermally conducting compound at the interface between the component and heat sink. A conventional heat sink model with grounded metal heat sink (EMC challenging model, standard problem 2000-4) and a new model with insulated metal heat sink (Intel P4 and AMD) are analyzed in the RF domain. A frequency domain approach technique is used in the simulation.

\section{Full WaVe Modeling Techniques}

To obtain a full solution to Maxwell's equations, for a structure of arbitrary size, a full-wave technique is required. A fullwave computational technique provides a complete solution to Maxwell's equations within the computational space for all conductors and materials. Full wave techniques are more complex than quasi-static techniques, but they are also generic in nature and have fewer limitations in their use.

Given the various full-wave techniques, each technique has its own strengths, and weaknesses. The limitations of practical fullwave techniques vary from technique to technique and on the level of detail required in the constructed model. Each full-wave

Digital Object Identifier 10.1109/TMAG.2006.880092 modeling technique is limited to particular types of models. Currently there is no single numerical technique that will solve all modeling problems that an EMC engineer is likely to encounter. The type of numerical technique chosen depends on the application problem.

The solution to a problem can be found in either the frequency or time domain. Solutions obtained in the time domain use a Fourier transform to provide output data as a function of frequency [1]. Since frequency domain codes must be run for each frequency of interest, sometimes a frequency sweeping technique is required for obtaining the solution in a certain range of frequencies [2], [3]. One major limitation of frequency domain method is the frequency range over which the model is valid. This limitation is primarily imposed by how finely the problem is partitioned and how close the elements are to the edges of the computational space. The computation error can be introduced if excitation sources extend beyond the valid frequency range.

\section{A. Time-Domain Techniques}

Time-domain techniques use a band-limited impulse to generate a wide frequency range excitation source as an input to the simulation. The result obtained from a time-domain code is the model's response to this impulse. A Fourier transform is applied to the time-domain data when frequency-domain information is required. While there are a large number of driving wave forms available, the most common waveform is in the form of a Gaussian pulse. A simple Gaussian pulse contains energy from direct current (dc) to a defined upper frequency. Another form is the differentiated Gaussian pulse that contains no dc component and has a 6-dB/oct falling value with decreasing frequency and the normal fast roll-off at high frequencies [2].

\section{B. Frequency-Domain Techniques}

Frequency-domain codes solve for one frequency at a time. This is usually adequate for antenna work and for examining specific issues. Frequency-domain codes are in general faster than their time-domain cousins. Therefore, several frequencydomain simulations can usually be run in the time it would take for a single time-domain simulation. A further benefit to 
using frequency-domain codes is their capacity to use larger meshes for the lower frequencies, which in turn permits a shorter computation time. To cover a wide frequency range with frequency-domain codes, a number of simulations are required. It should be noted that there are interpolation techniques available that minimize the number of simulations required. However, these interpolation techniques must be used with care to ensure a resonance effect is not omitted.

\section{EMC MODELING FOR CPU HEAT SINK}

The power generated from processor currents can reach levels of $50 \mathrm{~W}$. Moreover, clocking speeds are over $1 \mathrm{GHz}$. This combination of switching frequency and power level in conjunction with the layout of the common mode current paths through the heat sinks results in significant radiated EMI. As a result, EMC engineers need to understand the cause of radiated emissions from heat sinks, and find ways to reduce them whenever possible.

A joint IEEE/EMC Society Technical Committee (TC-9) and Applied Computational Electromagnetic Society (ACEM) have partnered in an effort to develop specific standard problems [4]. The CPU heat sink problem is one of the EMC challenging problems. The purpose of these problems is to provide EMI/EMC engineers and vendors with a way of determining the validity and accuracy of their EM modeling software. To model the heat sink structure, it is useful to break down the structure into the following three regions; the ground plane, the source region and the heat sink. A realistic representation of a VLSI must take into account the electromagnetic source characteristics and an actual physical model, e.g., a conducting patch [1]. Real heat sinks have fins to increase the thermal conductance. Brench [5] found that the heat sink could be modeled as a solid block. One approach from Das and Roy [6] models the source as a monopole that cuts through a VLSI. Das and Roy tested three cases: ground plane monopole, ground plane monopole through a VLSI and element through a VLSI with a heat sink. From the experimental results, Das and Roy concluded that as a first approximation, a monopole could be used to model the characteristics of a heat sink structure.

The Intel P4 processor in the 478-Pin Package has a different packaging and structural configuration compared with the conventional CPU configurations, as shown in Fig. 1. In the Intel P4 configuration, a heat spreader is located on top of the VLSI and the heat spreader is isolated from the VLSI packaging. Therefore, a different numerical model needs to be employed in contrast to the model used for the conventional CPU configurations and the IEEE challenging model, e.g., 2000-4. A simplified model consisting of a multi layered structure for the P4 and heat sink system is used, as shown in Fig. 2 [7]. The Intel Pentium 4 Processor is placed in a 478-Pin Package and can operate at $1.40,1.50,1.60,1.70,1.80,1.90$, and $2 \mathrm{GHz}[8]$.

\section{Computation Results of Intel P4 CPU Heat SinK}

The computer model of the Intel P4 CPU heat sink shown in Fig. 2 was simulated using a commercial available EM simulator; High Frequency Structure Simulator (HFSS) [9], which employs a finite-element frequency domain method. In



Fig. 1. Configuration of Intel P4 CPU [8]. (Color version available online at http://ieeexplore.ieee.org.)

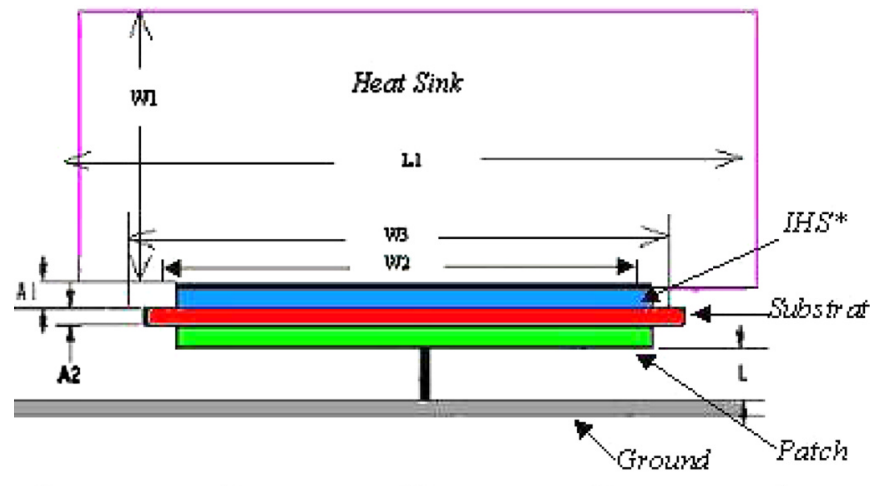

\begin{tabular}{|c|c|c|c|}
\hline Code Letter & $\operatorname{Min}(\mathrm{mm})$ & Neamal (mm) & $\operatorname{Mex}(\mathrm{mm})$ \\
\hline$A 1$ & 2.266 & 2.578 & 2.490 \\
\hline A2 & 0.980 & 1.080 & 1.180 \\
\hline 1 & 1.950 & 2.030 & 2.110 \\
\hline Ll & - & 88.9 & - \\
\hline W] &  & 381 &. \\
\hline $\mathrm{W} 2$ & $3 \mathrm{i}$ & 3175 & 32 \\
\hline $10 / 3$ & 349 & 35 & 351 \\
\hline \multicolumn{4}{|c|}{ - Bitegroved Beor Sithk } \\
\hline
\end{tabular}

Fig. 2. Computer simulation model of Intel P4 CPU and heat sink, where the following materials are used; IHS: copper, Substrate: polyamide, Patch: copper, Heat sink: aluminum, Ground plane: copper. (Color version available online at http://ieeexplore.ieee.org.)

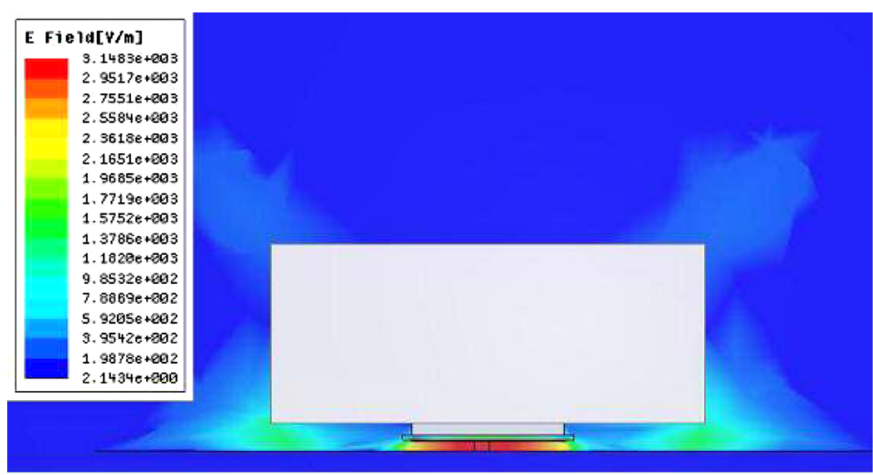

Fig. 3. The side view of the radiation energy flow observed from Intel P4 CPU heat sink simulation model with finite ground plane. (Color version available online at http://ieeexplore.ieee.org.)

this computer model, a radiation boundary is used to simulate an open problem that allows waves to radiate infinitely far into space. A radiation surface does not have to be spherical, but it must be 1) exposed to the background, 2) convex with regard to the radiation source, and 3 ) located at least a quarter wavelength from the radiating source. The shape of the boundary in our 


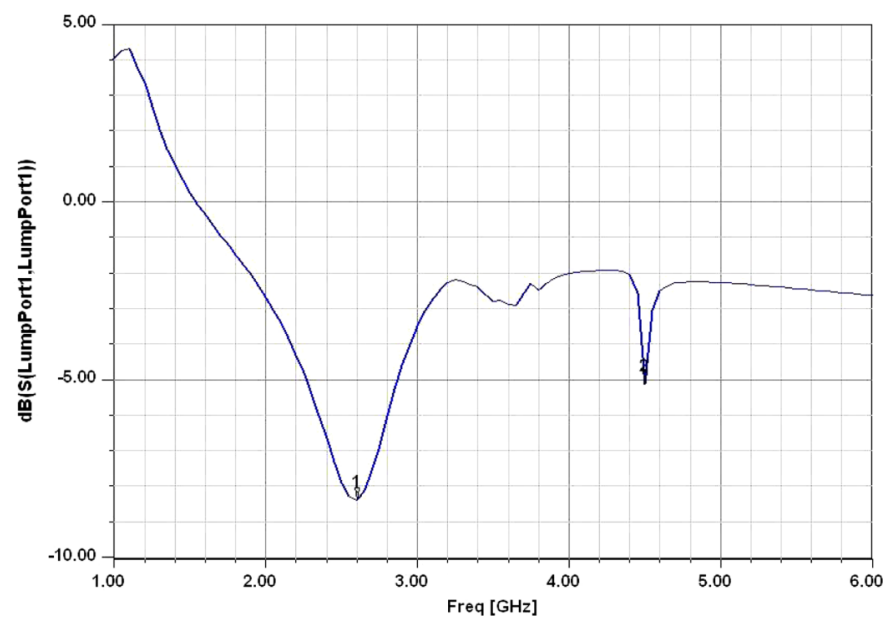

Fig. 4. Frequency sweeping results for P4 CPU heat sink model. (Color version available online at http://ieeexplore.ieee.org.)

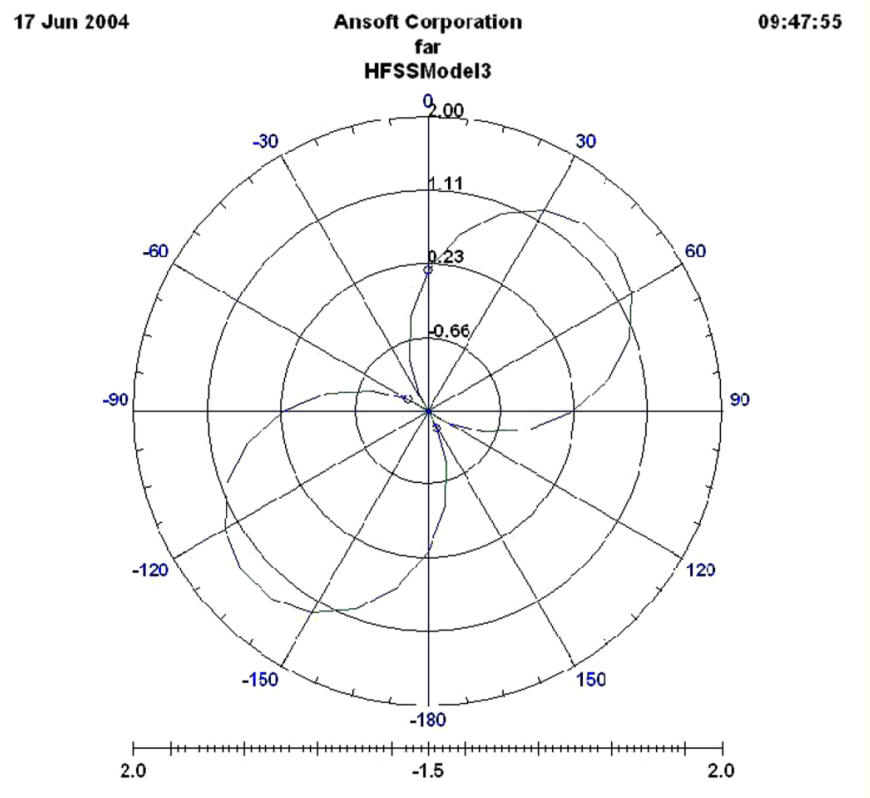

Fig. 5. P4 heat sink radiation pattern at $2.6 \mathrm{GHz}$ for the $\mathrm{E}$ theta field, where theta is equal to 0 degree. (Color version available online at http://ieeexplore.ieee.org.)

simulation model is spherical. The space in the sphere is the computational domain which is related to the frequency range we are interested in. The exciting port we used in our simulation is a Lumped port [9], where the excitation is a vertical source extending from the ground plane to the base of the conducting patch. It is electromagnetically coupled with the heat sink through the substrate. There is no grounding point for this $\mathrm{P} 4$ CPU heat sink model. Fig. 3 illustrates radiated energy flowing through the coupled patch to the heat sink and free space.

Fig. 4 shows the swept frequency results, where two resonant frequencies were found at 2.6 and $4.5 \mathrm{GHz}$, respectively. From the reflection coefficient, $\mathrm{S}_{11}$ at $2.6 \mathrm{GHz}$ we can predict that Intel P4 CPU heat sink worked as a perfect antenna and, thus, radiates energy to free space.

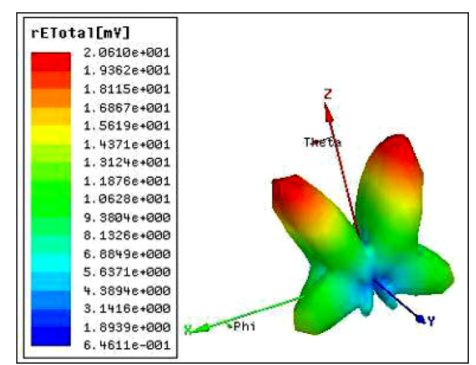

Fig. 6. Three-dimensional radiation pattern for $\mathrm{E}$ field emitted from $\mathrm{CPU}$ heat sink at $2.6 \mathrm{GHz}$. (Color version available online at http://ieeexplore.ieee.org.)

Fig. 5 illustrates the far field radiation pattern for the E field radiated from the Intel P4 CPU heat sink at $2.6 \mathrm{GHz}$. One can easily observe that there is a significant amount of radiated emission from this heat sink at $2.6 \mathrm{GHz}$.

Fig. 6 shows the three-dimensional far field radiation pattern for the E field at $2.6 \mathrm{GHz}$. There are two radiation beams in the $Z$ direction, which means the beam direction is more directional. The calculated antenna gain is larger than $>8 \mathrm{dBi}$, which is higher than most of the antennas for portable mobile devices in wireless communication systems.

\section{CONCLUSION}

This paper presented a finite-element frequency domain method to model the radiated emissions from a CPU heat sink. Comparing the results with the EMC Challenge model or the traditional model using a grounded heat sink configuration, the P4 CPU heat sink with an insulated configuration was found to radiate emission at $2.6 \mathrm{GHz}$. The second resonant frequency was found to be at $4.5 \mathrm{GHz}$. These resonant frequencies, within the wireless computing frequency range, are associated with the heat sink structure and the CPU clock speed. The future work is to reduce the radiated emission from the heat sink by optimizing the physical size of the heat sink and insulation structure.

\section{REFERENCES}

[1] K. Li et al., "Application of FD-TD method to analysis of EM radiation from VLSI configurations," IEEE Trans. Electromagn.Compat., vol. 35 , no. 2, pp. 204-213, May 1993.

[2] EMI/EMC Computational Modeling Hand Book, 2nd ed., Kluwer Academic, Norwell, MA, 2001. B. Archambeault, C. Brench, O. M. Ramahi

[3] P. Zhou and J. Lu, EMC Computer Modeling. Beijing, China: China Electric Power Press, Feb. 2005

[4] IEEE/EMC TC-9 and ACEM [Online]. Available: http://aces.ee.olemiss.edu/

[5] C. E. Brench, "Heatsink radiation as a function of geometry," in Proc. IEEE Symp. Electromagnetic Compatability, 1994, pp. 105-109.

[6] S. K. Das and T. Roy, "An investigation of radiated emissions from heatsinks," in Proc. IEEE Symp. Electromagnetic Compatability, vol. 2, 1998, pp. 784-789.

[7] J. Lu and D. Xiao, "EMC Computer Modeling Techniques for CPU Heat Sink Simulation," in Proc ICCEA2004, Dec. 2004, pp. 272-275.

[8] Intel Pentium 4 Processor in the 478-Pin Package, Apr. 2002. Intel Corporation, p. 47.

[9] HFSS v9.0 (2004). [Online]. Available: http://www.ansoft.com/products/hf/hfss/

Manuscript received March 3, 2006; revised June 10, 2006 (e-mail: j.lu@ griffith.edu.au). 